\title{
Association of the POT1 Germline Missense Variant p.I78T With Familial Melanoma
}

Kim Wong, MSc; Carla Daniela Robles-Espinoza, PhD; David Rodriguez, PhD; Saskia S. Rudat, PhD; Susana Puig, MD, PhD; Miriam Potrony, PhD; Chi C. Wong, MD, PhD; James Hewinson, PhD; Paula Aguilera, MD, PhD; Joan Anton Puig-Butille, PhD; Brigitte Bressac-de Paillerets, PharmD, PhD; Hélène Zattara, MD; Louise van der Weyden, PhD; Christopher D. M. Fletcher, MBBS, PhD;

Thomas Brenn, MD, PhD; Mark J. Arends, MD, PhD; Víctor Quesada, PhD; Julia A. Newton-Bishop, MD, PhD; Carlos Lopez-Otin, PhD; D. Timothy Bishop, PhD; Paul W. Harms, MD, PhD; Timothy M. Johnson, MD, PhD; Alison B. Durham, PhD; David B. Lombard, MD, PhD; David J. Adams, PhD

IMPORTANCE The protection of telomeres 1 protein (POT1) is a critical component of the shelterin complex, a multiple-protein machine that regulates telomere length and protects telomere ends. Germline variants in POT1 have been linked to familial melanoma, and somatic mutations are associated with a range of cancers including cutaneous T-cell lymphoma (CTCL).

OBJECTIVE To characterize pathogenic variation in POT1 in families with melanoma to inform clinical management.

DESIGN, SETTING, AND PARTICIPANTS In this case study and pedigree evaluation, analysis of the pedigree of 1 patient with melanoma revealed a novel germline POT1 variant (p.I78T, c.233T >C, chromosome 7, g.124870933A>G, GRCh38) that was subsequently found in 2 other pedigrees obtained from the GenoMEL Consortium.

MAIN OUTCOMES AND MEASURES (1) Identification of the POT1 p.I78T variant; (2) evaluation of the clinical features and characteristics of patients with this variant; (3) analysis of 3 pedigrees; (4) genomewide single-nucleotide polymorphism genotyping of germline DNA; and (5) a somatic genetic analysis of available nevi and 1 melanoma lesion.

RESULTS The POT1 p.I78T variant was found in 3 melanoma pedigrees, all of persons who self-reported as being of Jewish descent, and was shown to disrupt POT1-telomere binding. A UV mutation signature was associated with nevus and melanoma formation in POT1 variant carriers, and somatic mutations in driver genes such as BRAF, NRAS, and KIT were associated with lesion development in these patients.

CONCLUSIONS AND RELEVANCE POT1 p. I78T is a newly identified, likely pathogenic, variant meriting screening for in families with melanoma after more common predisposition genes such as CDKN2A have been excluded. It could also be included as part of gene panel testing.

Author Affiliations: Author affiliations are listed at the end of this article.

Corresponding Author: David J. Adams, PhD, Experimental Cancer Genetics, Wellcome Sanger Institute, Hinxton, Cambridge, CB10 1HH, England (da1@sanger.ac.uk). 
A bout $10 \%$ of patients with melanoma have a family history of the disease. ${ }^{1,2}$ The major melanoma predisposition genes are $C D K N 2 A, C D K 4$, and $B A P 1 .^{1} \mathrm{Ex}-$ tremely rare germline variants in the TERT promoter have also been reported. ${ }^{3,4}$ More recently, components of the shelterin complex, including in the Protection of Telomeres 1 (POT1) gene, $A C D$, and TERF2IP,,$^{5-7}$ have been implicated. Pathogenic $P O T 1$ variants promote telomere lengthening and disrupt telomere stability. ${ }^{5,6}$ Importantly, these variants are rare and potentially contribute to $1 \%$ to $5 \%$ of familial melanoma cases. ${ }^{5,6}$ Pathogenic POT1 variants have also been associated with predisposition to chronic lymphocytic leukemia (CLL), cardiac angiosarcoma, glioma, and, more recently, colon cancer. $^{8}$

\section{Methods}

Institutional review board approval for this study was obtained from the University of Leeds, University of Michigan, Hospital Clínic de Barcelona, and Gustave Roussy. All patients included in this study signed informed consent. Exome capture was performed using the SureSelect XT Human AllExon V5 platform (Agilent Technologies Inc), and sequencing

\section{Key Points}

Question What is the association of the p.I78T variant in the Protection of Telomeres 1 gene (POT1) with familial melanoma?

Findings We identified the POT1 p.I78T variant in 3 families of Jewish heritage with melanoma in the family and provide evidence that p.178T interferes with POT1-telomere binding, a critical disruption associated with tumor predisposition. These findings suggest that the POT1 p.178T variant may represent a founder allele.

Meaning The identification of high-penetrance germline variants such as POT1 p.178T, facilitates screening and counseling of at-risk patients.

was performed using the Illumina HiSeq 2000 platform (Illumina Inc). Telomere binding and length assays were performed as previously described. ${ }^{5}$ For analysis methods, see the Supplement.

\section{Results}

Our analysis began with the identification of a North American proband who had developed primary melanoma at age

Figure 1. Lesion Locations and 3 Pedigrees of Familial Melanoma, Including the Proband's (Pedigree 1)
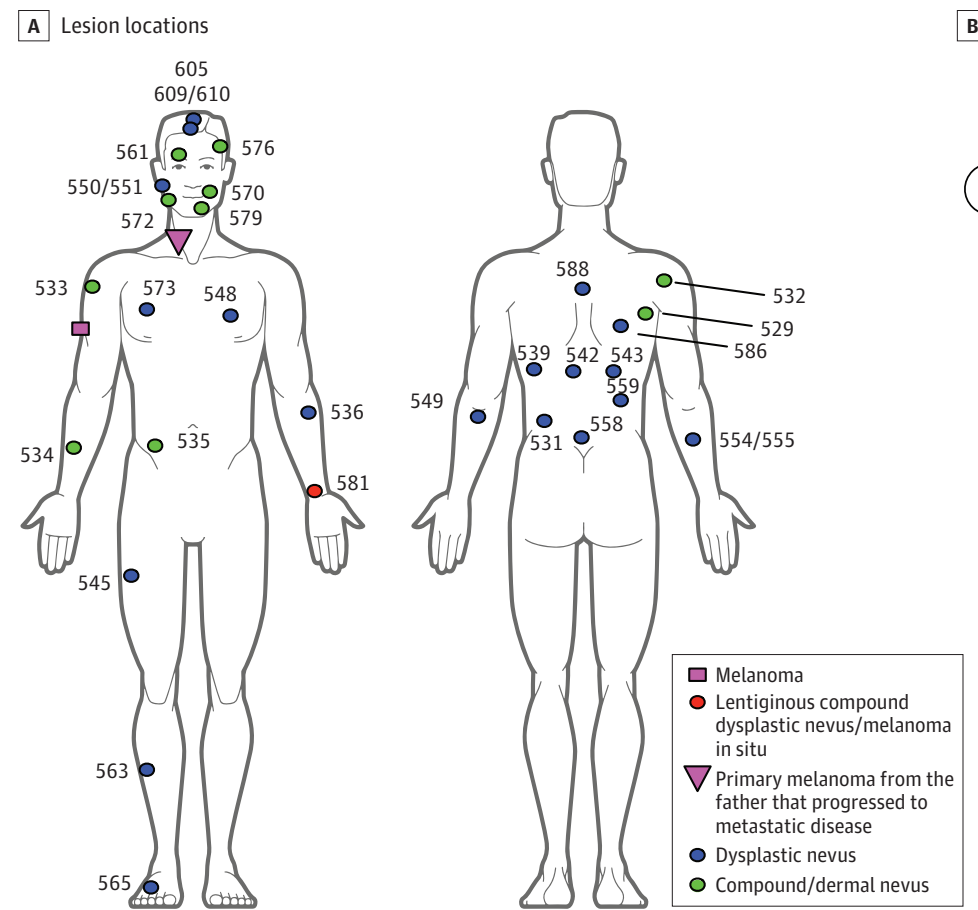

B Melanoma family pedigrees
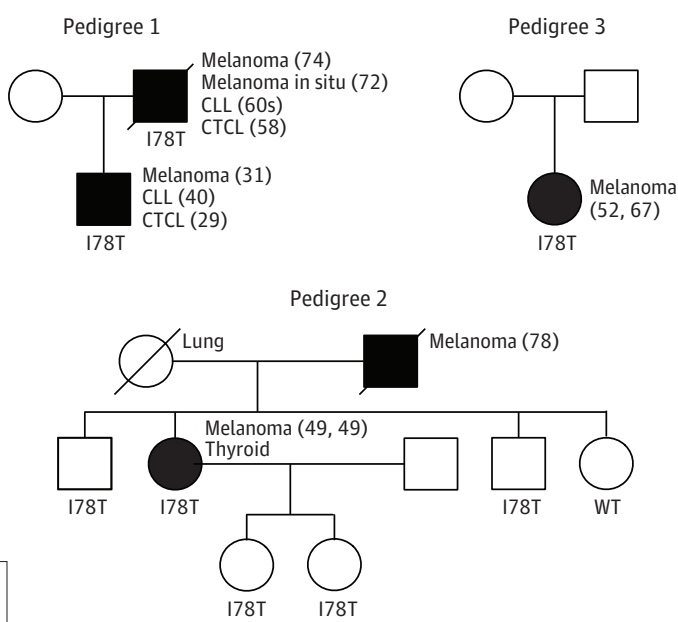

In this proband and all 3 family pedigrees, the POT1 p.I78T variant was identified as the likely pathogenic variant; all 3 pedigrees show the same base change. A, Locations of lesions identified and excised from the proband, a POT1 variant carrier (p.178T, c.233T>C, chromosome 7, g.124870933A>G, GRCh38) at age 31 years. The primary melanoma on the neck was from the proband's father, not the proband, but is added here to illustrate its position. This melanoma progressed to metastatic disease in the father. All numbered lesions were sequenced. Samples numbered 550/551, 554/555, and 609/610 indicate 2

samples taken from the same lesion in these 3 cases. B. Pedigree structures for all families identified to carry the p.I78T variant. Pedigree 1 corresponds to that of the proband, and pedigrees 2 and 3 were identified from the GenoMEL Consortium. Individuals represented by black shapes are variant carriers who presented with melanoma. Individuals with no genotype were not tested. Ages, reported in years, at the times of cancer diagnoses are shown in parentheses when available. 
Figure 2. Functional Analyses of the Pathogenicity of the POT1 p.I78T Variant

A Multispecies POT1 protein sequence alignment

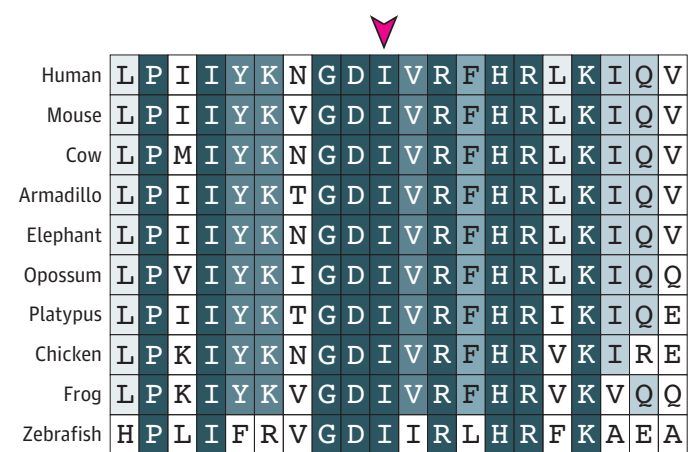

C Telomere gel shift assays

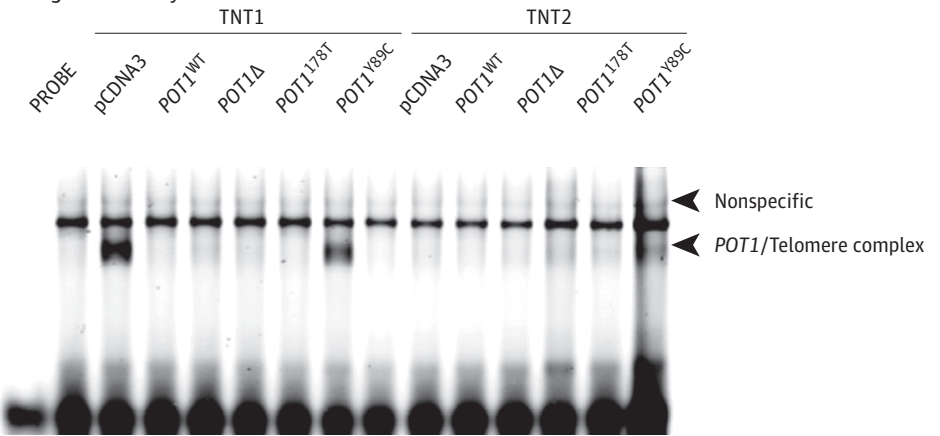

B Protein locations in the POT1 OB domain

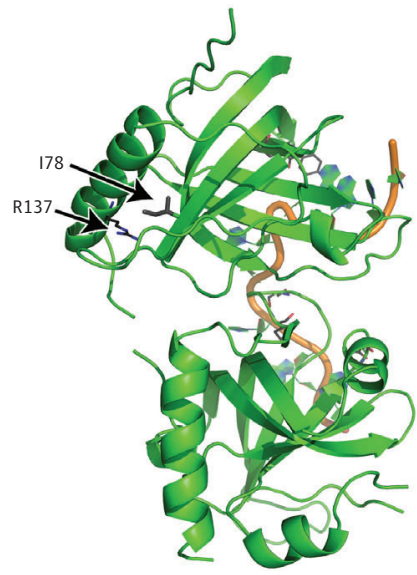

A, Multiple sequence alignment of the protection of telomeres 1 (POT1) protein sequence in humans and 9 other species. Shown are amino acid positions 69 to 88 , relative to human POT1, with the red arrow indicating the highly conserved 178 residue. The best match in each protein alignment relative to human protein is shown. $\mathrm{B}$, The location of the 178 residue on the structure of the POT1 oligonucleotide/oligosaccharide-binding fold (OB) domain is indicated, alongside another residue previously described as mutated in melanoma families (R137). Green ribbons represent the OB domains; orange thread, the telomere-like sequence. C, Telomere gel shift assays show that the POT1 p.I78T protein is unable to bind to a telomere-like sequence. Wild-type (WT) POT1 POT1 Y89C (a known pathogenic/disruptive variant) and POT1 (OB domain deletion) constructs were used as controls. ${ }^{5}$ TNT1 and TNT2 indicate separate in vitro translation reactions; pcDNA3 is an empty vector control. The arrow indicates the presence of a POT1 protein-telomere complex formed by the binding of in vitro translated POT1 protein to a telomere-like probe. Disruption of this protein-DNA complex is associated with telomere instability and a loss of telomere length control.
31 years along with multiple dysplastic and compound and dermal nevi (Figure 1A). He had also developed cutaneous T-cell lymphoma (CTCL) and CLL and at ages 29 and 40 years, respectively. His father had developed CTCL, CLL, melanoma in situ, and melanoma at ages 58, early 60s, 72 , and 74 years, respectively (Figure 1B, pedigree 1). The proband had sequenced his own genome on a nextgeneration platform and had identified a POT1 variant (p.I78T, c.233T>C, chromosome 7, g.124870933A>G, GRCh38), the pathogenicity of which was unknown, but it was predicted to be damaging by SIFT (score 0$)^{9}$ and Polyphen-2 (score 1.0) ${ }^{10}$ analysis. To determine the frequency of this variant in melanoma families, we ascertained cases by consulting The Melanoma Genetics Consortium (GenoMEL, http://www.genomel.org) and found 2 more carrier families; one of Spanish ancestry (1 case among 171 families) (Figure 1B, pedigree 2; more detail on the Spanish pedigree will be published elsewhere) and the other of French descent ${ }^{6}$ ( 1 case among 157 families)
(Figure 1B, pedigree 3), both of whom had tested negative for other high-penetrance genes (eMethods in the Supplement).

We next investigated whether the p.I78T variant had a functional effect. An analysis of orthologous protein sequences spanning 450 million years of evolutionary distance showed complete conservation of the isoleucine at position 78 (Figure 2A), and the protein model showed that this residue is in close proximity to arginine 137 , another position found to be mutated in melanoma pedigrees (Figure 2B). ${ }^{6}$ This suggested that alteration of this residue might impair POT1 single-stranded DNA binding. To test this, we generated a POT1 complementary DNA (cDNA) construct encoding the POT1 p.I78T protein and found that it was severely impaired for its ability to bind to a polynucleotide telomere-like probe (IR700GGTTAGGGTTAGGGTTAGGG) (Figure 2C), as previously seen with established melanoma-predisposing alleles. ${ }^{5}$ To provide further functional proof, we transduced 
telomerase-positive HT1080 cells with either wild-type or p.I78T POT1 cDNA constructs and cultured them through 45 population doublings. Telomeres in cells expressing the p.I78T POT1 cDNA were elongated compared with cells expressing wild-type POT1 (eMethods and eFigure 1 in the Supplement).

After identifying 3 apparently unrelated families living in different countries carrying the POT1 p.I78T variant, we genotyped individuals using CoreExome single-nucleotide polymorphism arrays (Illumina Inc) to estimate the coefficient of relatedness between carriers of the variant from different families and performed an analysis that suggested no recent co-ancestry between the families. We were also able to confirm the reported genetic relationships within each family. Although the families were not closely related, the sharing of genotypes around the POT1 gene in p.I78T variant carriers was consistent with a common haplotype across some 4 megabase pairs ( $\mathrm{Mb})$ upstream and downstream of the variant, suggesting that the p.I78T variant is a founder event.

Interestingly, all 3 families self-reported being Jewish, and we noted that the chromosome 7 g.124870933A>G change has been reported in 3 individuals of Ashkenazi Jewish descent in the Genome Aggregation Database (gnomAD; chromosome 7 g.124510987A $>$ G in GRCh37; allele frequency in the Ashkenazi Jewish population, 0.0003105; in all other populations, 0.000004305). ${ }^{11}$ Since patients with germline POT1 variants are highly predisposed to melanoma development, we wondered if there would be any defining features of the somatic landscape of melanocytic lesions in these patients. Thus, we whole-exome sequenced 30 previously excised, formalin-fixed, paraffin-embedded nevi from the proband in pedigree 1 (Figure 1) and a large malignant melanoma from the proband's father (anatomic location illustrated in Figure 1A; eMethods in the Supplement). Hotspot NRAS (Q61L, Q61K and Q61R) and BRAF (V600E, V600D) mutations were identified in nevi, and the melanoma was found to be KIT L576P positive and to carry truncating NF1 and RASA2 mutations (eTable 1 in the Supplement), suggesting a prominent role for the MAPK pathway. No TERT promoter mutations were found.

Since a major driver of melanoma formation is exposure to UV light, we next attempted to identify the imprint of this mutagen on the melanoma genome. This analysis revealed an average of 40.67 somatically acquired mutations per $\mathrm{Mb}$ of exome, of which $87 \%$ were $\mathrm{C}>\mathrm{T} / \mathrm{G}>\mathrm{A}$ mutations. Extraction of mutation signatures from the melanoma and nevi mutation catalogs identified the UV-associated signature 7 (cosine similarity, 0.97). ${ }^{12}$ The number of mutations we identified in the father's melanoma (Figure 1B, pedigree 1) was higher than the average observed following an analysis of 318 melanomas (16.8 mutations/Mb) sequenced by The Cancer Genome Atlas (TCGA), ${ }^{13}$ suggesting a contribution from UV light to melanomagenesis in this POT1 mutation carrier. The exome sequence of the CLL from the proband of pedigree 1 (Figure 1B) revealed few somatic mutations and no
Figure 3. Mutation Spectra of Tumors From POT1 p.I78T Carriers From Pedigree 1

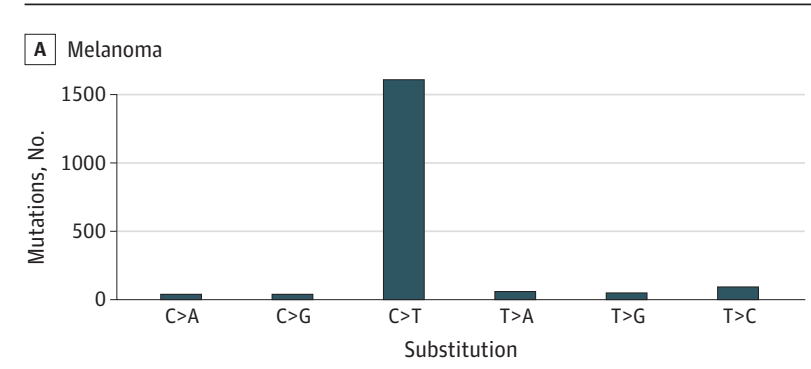

B Chronic lymphocytic leukemia

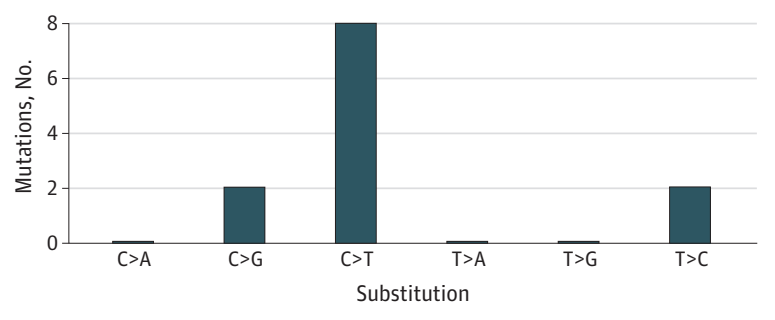

The top panel shows the exome-wide mutational spectra of the melanoma from the father, and the bottom panel shows the exome-wide mutation spectra from the proband's chronic lymphocytic leukemia.

enrichment for $\mathrm{C}>\mathrm{T}$ mutations (Figure 3; eTable 2 in the Supplement). All of the carriers in pedigrees 2 and 3 (Figure 1B) who had presented with melanoma had been diagnosed with either superficial spreading or nodular cutaneous melanoma. A histopathological analysis of the melanoma from the p.I78T variant carrier (father, pedigree 1 , Figure 1B) revealed that there were no defining features distinguishable from sporadic melanoma (eFigure 2 in the Supplement).

\section{Discussion}

The identification of pathogenic germline variants is of critical importance because it informs genetic counselling and allows individuals at high risk to be prioritized for surveillance programs. In this study, we identified the POT1 p.I78T variant in 3 unrelated melanoma pedigrees, with evidence for the presence of this allele at a higher frequency among Ashkenazi Jews. Interestingly, only 1 founder allele (p.V59G; CDKN2A) has previously been associated with melanoma risk in people of Jewish heritage. ${ }^{14}$

Notably, as illustrated in Figure 1B, in addition to melanoma, members of pedigree 1 have been diagnosed with both CTCL and CLL, while pedigree 2 contains a variant carrier who developed melanoma and thyroid cancer. Collectively, this clinical picture may suggest that this variant contributes to a range of cancers. Indeed, somatic POT1 p.I78T mutations have been found in a case of ovarian cancer (COSMIC ID: COSM116403) and in a case of hepatosplenic T-cell lymphoma. ${ }^{15}$ 


\section{Limitations}

Our study ascertained cases from GenoMEL, meaning that we may not have a full picture of the spectrum of cancers acquired by variant carriers. Additionally, while 4 of the 8 variant carriers illustrated in Figure 1B have developed melanoma, 4 variant carriers in pedigree 2 have not yet presented with melanoma or any other malignancy, and these individuals were 19, 25, 50, and 55 years old, respectively, at last followup. Thus, the penetrance of melanoma and other cancers in p.I78T variant carriers is yet to be defined. Ultimately the identification of further pedigrees with the p.I78T variant will help define its role in tumorigenesis.

\section{Conclusions}

The germline POT1 p.I78T variant is a candidate predisposition allele for melanoma development because it disrupts POT1-telomere binding and promotes telomere lengthening. Screening for this variant could be considered in individuals found to be negative for pathogenic variants in established melanoma predisposition genes such as CDKN2A, BAP1, and $C D K 4$, particularly in families of Jewish heritage and/or with additional POT1-associated cancers such as CTCL and CLL. ${ }^{16,17}$

\section{ARTICLE INFORMATION}

Accepted for Publication: August 23, 2018.

Published Online: December 26, 2018. doi:10.1001/jamadermatol.2018.3662

Author Affiliations: Experimental Cancer Genetics, The Wellcome Trust Sanger Institute, Hinxton, England (K. Wong, Robles-Espinoza, Rudat, C. C. Wong, Hewinson, van der Weyden, Adams); Laboratorio Internacional de Investigación sobre el Genoma Humano, Universidad Nacional Autónoma de México, Campus Juriquilla, Santiago de Querétaro, Qro, Mexico (Robles-Espinoza); Departamento de Bioquímica y Biología Molecular, Instituto Universitario de Oncología del Principado de Asturias (IUOPA), Universidad de Oviedo, Oviedo, Spain (Rodriguez, Quesada, Lopez-Otin); Centro de Investigación en Red de Cáncer (CIBERONC), Madrid, Spain (Rodriguez, Quesada, Lopez-Otin); Melanoma Unit, Department of Dermatology, Hospital Clínic de Barcelona, IDIBAPS Barcelona University, Barcelona, Spain (Puig Potrony, Aguilera); Centre of Biomedical Research on Rare Diseases (CIBERER), ISCIII, Barcelona, Spain (Puig, Potrony, Aguilera, Puig-Butille); Biochemistry and Molecular Genetics Department, Melanoma Unit, Hospital Clínic de Barcelona, IDIBAPS Barcelona University, Barcelona, Spain (Puig-Butille); Gustave Roussy, Université Paris-Saclay, Département de Biologie et Pathologie Médicales, Villejuif, France (Bressac-de Paillerets) INSERM U1186, Université Paris-Saclay, Villejuif, France (Bressac-de Paillerets); Département de Génétique, APHM, CHU Timone-Enfants, Marseille, France (Zattara); Department of Pathology Brigham \& Women's Hospital, Boston Massachusetts (Fletcher); Pathology Department, Western General Hospital, Edinburgh, Scotland (Brenn, Arends); Section of Epidemiology and Biostatistics, Leeds Institute of Cancer and Pathology, University of Leeds, Leeds, England (Newton-Bishop, Bishop); Department of Pathology, University of Michigan, Ann Arbor (Harms, Lombard); Department of Dermatology, University of Michigan, Ann Arbor (Johnson, Durham).

Author Contributions: Ms K. Wong and Dr Robles-Espinoza contributed equally to this work. Dr Adams had full access to all the data in the study and takes responsibility for the integrity of the data and the accuracy of the data analysis. Concept and design: Robles-Espinoza, Bishop, Lombard, Adams. Acquisition, analysis, or interpretation of data: All authors.

Drafting of the manuscript: K. Wong
Robles-Espinoza, Bishop, C. Wong, Hewinson, Adams.

Critical revision of the manuscript for important intellectual content: $\mathrm{K}$. Wong, Robles-Espinoza, Rodriguez, Puig, Potrony, Aguilera, Puig-Butillé, Bressac-de Paillerets, Zattara, van der Weyden, Fletcher, Brenn, Arends, Newton-Bishop, Quesada, Lopez-Otin, Bishop, Lombard, Harms, Adams. Statistical analysis: Quesada, Bishop. Obtained funding: Puig-Butillé, Zattara, Newton -Bishop, Bishop, Adams.

Administrative, technical, or material support: Rodriguez, Bressac-de Paillerets, van der Weyden, Fletcher, Brenn, Newton -Bishop, Lopez-Otin Bishop, Harms, C. Wong, Hewinson, Adams. Supervision: Aguilera, Adams.

Conflict of Interest Disclosures: None reported.

Funding/Support: The work was funded by Cancer Research UK (Program grant C588/A19167 and clinical research fellowship grant C37059/A17894) and the Wellcome Trust. The study in the Melanoma Unit, Hospital Clínic, Barcelona was supported in part by grants from Fondo de Investigaciones Sanitarias P.I. 12/00840, PI15/ 00956 and 15/00716 Spain; by the CIBER de Enfermedades Raras of the Instituto de Salud Carlos III, Spain, co-funded by “Fondo Europeo de Desarrollo Regional (FEDER). Unión Europea. Una manera de hacer Europa"; by the AGAUR 2009 SGR 1337 and AGAUR 2014_SGR_603 of the Catalan Government, Spain; by a grant from "Fundació La Marató de TV3, 201331-30", Catalonia, Spain; by the European Commission under the 6th Framework Programme, Contract No.: LSHC-CT-2006-018702 (GenoMEL); by CERCA Programme/Generalitat de Catalunya and by a Research Grant from Asociación Española Contra el Cáncer. Miriam Potrony is the recipient of a PhD Fellowship FI14/O0231 (PFIS) from Instituto de Salud Carlos III, Spain. This work was also supported by grants from Ministerio de Economía y Competitividad; Instituto de Salud Carlos III: CIBERONC, Plan Feder and EDP Foundation. We also thank the generous support by J.I. Cabrera. The Instituto Universitario de Oncología is supported by Fundación Bancaria Caja de Ahorros de Asturias. Dr Robles-Espinoza is funded by a Wellcome Trust Seed Award in Science, 204562/Z/16/Z, a PAPIIT grant from the Universidad Nacional Autónoma de México, IA200318 and the "Miguel Alemán Valdés" Medical Research Stimulus, Fundación Miguel Alemán, A.C.

Role of the Funder/Sponsor: The funders/ sponsors had no role in the design and conduct of the study; collection, management, analysis, and interpretation of the data; preparation, review, or approval of the manuscript; and decision to submit the manuscript for publication.

Additional Contributions: We thank the patients and their families for granting permission to publish this study. We acknowledge the valuable contribution of Vincent Caumette, Catherine Gensollen, and Biobank BB-033-00074 for their work on pedigree 3 in Figure 1B. They received no compensation for their contributions.

\section{REFERENCES}

1. Hill VK, Gartner JJ, Samuels Y, Goldstein AM. The genetics of melanoma: recent advances. Annu Rev Genomics Hum Genet. 2013:14:257-279. doi:10. 1146/annurev-genom-091212-153429

2. Read J, Wadt KA, Hayward NK. Melanoma genetics. J Med Genet. 2016;53(1):1-14. doi:10.1136/ jmedgenet-2015-103150

3. Harland M, Petljak M, Robles-Espinoza CD, et al. Germline TERT promoter mutations are rare in familial melanoma. Fam Cancer. 2016;15(1):139-144 doi:10.1007/s10689-015-9841-9

4. Horn S, Figl A, Rachakonda PS, et al. TERT promoter mutations in familial and sporadic melanoma. Science. 2013;339(6122):959-961. doi: 10.1126/science.1230062

5. Robles-Espinoza CD, Harland M, Ramsay $A J$, et al. POT1 loss-of-function variants predispose to familial melanoma. Nat Genet. 2014;46(5):478-481 doi:10.1038/ng.2947

6. Shi J, Yang XR, Ballew B, et al; NCI DCEG Cancer Sequencing Working Group; NCI DCEG Cancer Genomics Research Laboratory; French Familial Melanoma Study Group. Rare missense variants in POT1 predispose to familial cutaneous malignant melanoma. Nat Genet. 2014:46(5):482-486. doi: 10.1038/ng.2941

7. Aoude LG, Pritchard AL, Robles-Espinoza CD, et al. Nonsense mutations in the shelterin complex genes ACD and TERF2IP in familial melanoma. J Natl Cancer Inst. 2014;107(2):dju408.

8. Calvete O, Garcia-Pavia P, Domínguez F, et al. The wide spectrum of POT1 gene variants correlates with multiple cancer types. Eur J Hum Genet. 2017; 25(11):1278-1281. doi:10.1038/ejhg.2017.134

9. Ng PC, Henikoff S. SIFT: predicting amino acid changes that affect protein function. Nucleic Acids Res. 2003;31(13):3812-3814. doi:10.1093/nar/gkg509

10. Adzhubei I, Jordan DM, Sunyaev SR. Predicting functional effect of human missense mutations using PolyPhen-2. Curr Protoc Hum Genet. 2013; Chapter 7:20. doi:10.1002/0471142905.hg0720s76 
11. Lek M, Karczewski KJ, Minikel EV, et al; Exome Aggregation Consortium. Analysis of protein-coding genetic variation in 60,706 humans. Nature. 2016;536(7616):285-291. doi:10.1038/ nature19057

12. Alexandrov LB, Nik-Zainal $S$, Wedge DC, et al; Australian Pancreatic Cancer Genome Initiative; ICGC Breast Cancer Consortium; ICGC MMML-Seq Consortium; ICGC PedBrain. Signatures of mutational processes in human cancer. Nature. 2013:500(7463):415-421. doi:10.1038/nature12477
13. Cancer Genome Atlas Network. Genomic classification of cutaneous melanoma. Cell. 2015;161 (7):1681-1696. doi:10.1016/j.cell.2015.05.044

14. Yakobson $E$, Eisenberg $S$, Isacson $R$, et al. A single Mediterranean, possibly Jewish, origin for the Val59Gly CDKN2A mutation in four melanoma-prone families. Eur J Hum Genet. 2003; 11(4):288-296. doi:10.1038/sj.ejhg.5200961

15. McKinney M, Moffitt $A B$, Gaulard $P$, et al. The genetic basis of hepatosplenic T-cell lymphoma. Cancer Discov. 2017;7(4):369-379. doi:10.1158/ 2159-8290.CD-16-0330
16. Woollard WJ, Pullabhatla V Lorenc A, et al. Candidate driver genes involved in genome maintenance and DNA repair in Sézary syndrome. Blood. 2016;127(26):3387-3397. doi:10.1182/blood2016-02-699843

17. Pinzaru AM, Hom RA, Beal A, et al. Telomere replication stress induced by POT1 inactivation accelerates tumorigenesis. Cell Rep. 2016;15(10) 2170-2184. doi:10.1016/j.celrep.2016.05.008 